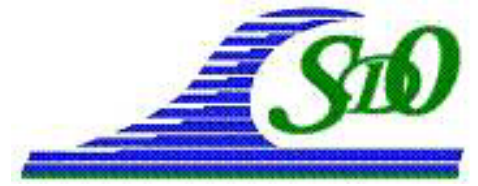

\title{
DRELIO : Un drone hélicoptère pour le suivi des zones littorales
}

\author{
Marion JAUD ${ }^{1}$, Christophe DELACOURT ${ }^{1}$, Pascal ALLEMAND ${ }^{2}$, \\ Philippe GRANDJEAN ${ }^{2}$, Jérôme AMMANN ${ }^{1}$, Romain CANCOUËT ${ }^{1}$, \\ Anne DESCHAMPS ${ }^{1}$, Eric VARREL ${ }^{3}$, Véronique CUQ ${ }^{4}$, Serge SUANEZ ${ }^{4}$
}

1 IUEM, Université de Bretagne Occidentale, CNRS UMR6538, 29280 Plouzané. marion.jaud@univ-brest.fr

2. Université de Lyon 1 et ENS-Lyon - CRS UMR5570, 69662 Villeurbanne.

3. ATM 3D.

4. IUEM, Université de Bretagne Occidentale, CNRS UMR6554, 29280 Plouzané.

\section{Résumé :}

La connaissance du littoral, sa protection, son aménagement nécessitent un suivi des changements qui s'y produisent. Toutefois, en France, devant l'étendue des façades maritimes à couvrir, les techniques de mesures in situ ne peuvent être appliquées de façon systématique. Dans ce cas, les techniques de télédétection spatiale ou aérienne sont des approches complémentaires. A partir de ces plates-formes, des images stéréoscopiques multi-temporelles sont, après une série de traitements photogrammétriques appropriés, directement exploitables sous formes d'orthophotographies et de Modèles Numériques de Terrain (MNT). Ce type de produits permet notamment la quantification des changements morphosédimentaires à l'interface Terre-Mer (transport transversal et longitudinal de sédiments, érosion,...). Actuellement, la résolution spatiale des MNT générés à partir d'images aériennes ou satellitaires $(<50 \mathrm{~cm})$ est insuffisante pour la plupart des applications sur la frange littorale. En outre, dans le cas d'interventions consécutives à un événement extrême (tempête, raz de marée, pollution...), ces systèmes manquent de souplesse (trajectoires prédéfinies, contraintes d'altitude, de vitesse, coût de mise en œuvre...) L'utilisation de drones constitue donc une alternative intéressante pour des suivis de précision ou des interventions rapides.

\section{Mots-clés :}

Télédétection - MNT - Drone - Stréréorestitution - Photogrammétrie - Suivi de plages

\section{Introduction}

Le suivi temporel du littoral est une étape incontournable pour anticiper les risques côtiers (RUGGIERO et al., 2000; RIEB \& WALKER, 2001) et comprendre les dynamiques physico-chimiques de cette zone d'interface. D'une part, ces suivis apportent des informations primordiales en termes de gestion littorale, pour la protection des côtes ou les plans d'occupation des sols (HAMM et al., 2002 ; MEUR- 
FEREC et al., 2008). D'autre part, les modèles numériques hydro- ou morphodynamiques à l'interface Terre-Mer requièrent des mesures in situ pour leur calibration et leur validation (SOLOMON \& FORBES, 1999). De par leur perception synoptique, les techniques de télédétection sont donc de plus en plus employées pour collecter les informations géométriques, cinématiques et dynamiques des domaines océaniques et côtiers (DEKKER et al., 2001).

Jusqu'à présent, les images satellitaires étaient privilégiées bien qu'elles présentent des limitations en termes de résolution spatiale restreinte malgré les progrès récents, de couverture nuageuse, ou bien d'heure locale d'acquisition fixe, ... . L'utilisation de plates-formes aéroportées résout en partie ces problèmes puisqu'elles permettent d'obtenir des données avec une résolution spatiale inférieure à $50 \mathrm{~cm}$ (CASSON et al., 2005). De plus, la télédétection aérienne permet l'acquisition de couples stéréoscopiques à partir desquels des Modèles Numériques de Terrain (MNT) de précision submétrique peuvent être calculés. En complément des images aériennes multi-temporelles qui permettent de déterminer les évolutions 2D du trait de côte, les MNT multi-temporels permettent un suivi 3D de la morphologie des plages. Néanmoins, le coût financier, humain et technique de telles missions sur des zones très ciblées peut paraître prohibitif. Pour lever ces dernières limitations, de nouvelles techniques sont mises en oeuvre. Des plates-formes de tailles réduites, telles que des cerf-volants, des ballons et des drones apparaissent dors et déjà comme des outils très prometteurs pour les levés topographiques de glissements de terrain ou d'érosion de pentes (CASSON et al., 2005 ; JACOME et al., 2009), de rivières (LEJOT et al., 2007), mais également pour la surveillance des cultures (SUGIURA et al., 2005). Ces plates-formes sont d'une utilisation très flexible et permettent, pour un coût modéré, d'effectuer des levés répétés à périodicité variable (hebdomadaire à annuelle). L'acquisition multi-temporelle d'images haute résolution permet de générer des MNT de résolution et précision centimétrique à décimétrique et des orthophotographies. Ces produits permettent ainsi l'observation des changements littoraux, la mesure de déplacements horizontaux et la réalisation de bilans de matière. Nous présenterons dans cet article le potentiel, les limites, ainsi qu'un exemple d'utilisation en Bretagne d'un hélicoptère radiocommandé développé par notre équipe pour l'imagerie côtière : DRELIO (DRone hELIcoptère pour l'Observation de l'environnement).

L'Institut Universitaire Européen de la Mer (IUEM) et l'Université Lyon 1 ont développé un hélicoptère photogrammétrique télécommandé : DRELIO (DELACOURT et al., 2009). Equipé d'un système d'auto-pilotage et pouvant voler jusqu'à $200 \mathrm{~m}$ d'altitude, DRELIO est également conçu pour exécuter des vols stationnaires et résister à des vents de $50 \mathrm{~km} / \mathrm{h}$. Il est équipé d'un appareil photographique réflex muni d'objectifs interchangeables et d'une caméra thermique. La résolution des images varie en fonction de l'altitude de vol et de la focale de l'objectif. A titre d'exemple, pour un vol à $100 \mathrm{~m}$ d'altitude et une focale de $35 \mathrm{~mm}$, la zone couverte est de $67 \times 45 \mathrm{~m}$ avec 
une résolution de $1,7 \mathrm{~cm} /$ pixel. Étant donnée la spécificité des conditions de vol et de prise de vue, une chaîne de traitement photogrammétrique a été développée. L'utilisation de clichés stéréoscopiques et de points de référence positionnés au GPS différentiel permet de générer des images orthorectifiées et des MNT avec une résolution de l'ordre de $5 \mathrm{~cm}$.

\section{DRELIO}

Le système DRELIO peut être décomposé en 4 unités majeures (voir figure 1):

a) un hélicoptère de $1,80 \mathrm{~m}$ de longueur et $2 \mathrm{~m}$ d'envergure,

b) un support tourelle pour les capteurs scientifiques (appareil photographique, caméra),

c) un système embarqué pour le contrôle du vol (pilote automatique, GPS, gyroscope, magnétomètre),

d) un système de contrôle au sol (ordinateur, manette de commande, radio).

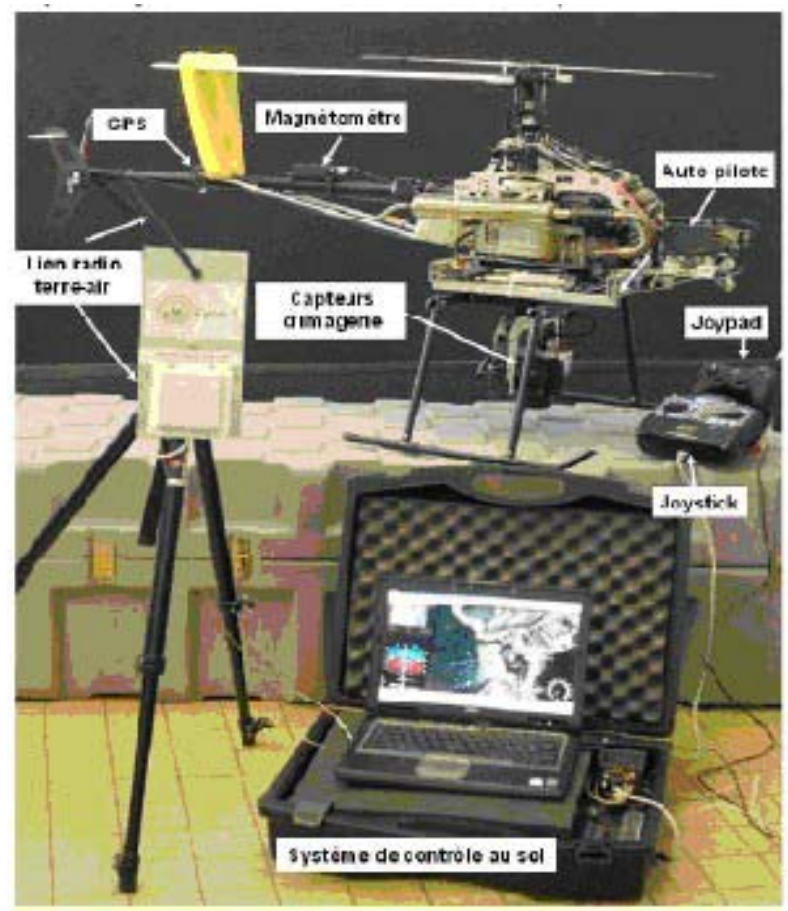

Figure 1. Configuration technique de DRELIO.

L'hélicoptère est un modèle thermique acrobatique Vario rigidifié. Pour adapter DRELIO au domaine marin, il a été protégé de la corrosion, par une couche de Teflon et toutes les connexions de câbles ont été isolées. Son poids à vide est de $11 \mathrm{~kg}$, autorisant une charge utile supplémentaire de $6 \mathrm{~kg}$. Afin de limiter les vibrations et d'augmenter la stabilité en vol, la vitesse de rotation des pales a été réduite. La vitesse maximale de DRELIO est de $70 \mathrm{~km} \mathrm{~h}^{-1}$ pour une autonomie d'environ d'une heure. Ce système est peu dépendant des conditions aérologiques, des missions ont été réalisées avec des vents 
supérieurs à 30 nœuds. Sous l'hélicoptère a été installée une tourelle rotative dont le mouvement est contrôlable en temps réel depuis le sol ou programmable avant la mission. Le vol peut être géré par un pilote automatique connecté à des capteurs de pression atmosphérique, un capteur inertiel, un capteur géomagnétique et un GPS. Une caméra vidéo basse résolution est fixée sur le viseur de l'appareil photo reflex numérique. Les images de la caméra sont transférées en temps réel au sol ce qui permet de contrôler les vues acquises en vol. L'appareil photo peut être remplacé par une caméra sensible à infrarouge thermique couplée à un appareil photographique numérique compact.

Le décollage de DRELIO peut être entièrement automatique. DRELIO peut ensuite suivre un plan de vol prédéfini et atterrir de façon autonome. Le drone étant équipé d'un GPS, il est possible de programmer à l'avance la position du drone pour le déclenchement des clichés afin d'effectuer des comparaisons multi-temporelles.

Le lien radio autorise des communications avec la base au sol jusqu'à $60 \mathrm{~km}$ de distance. Néanmoins, une fois le plan de vol chargé, toute communication avec le sol est superflue, ce qui augmente donc le rayon d'action. En fonction du mode d'utilisation du pilote automatique (manuel, stationnaire, vol planifié), il est possible de contrôler le drone à distance de manière manuelle ou semi-manuelle.

\section{Mission DRELIO type}

Afin d'optimiser les conditions de vol et de prises de vue, les missions sont préférentiellement planifiées avant le lever ou après le coucher du soleil. L'éclairement est alors homogène, les ombres limitées et les conditions aérologiques plus stables.

Toutefois, en domaine côtier, la programmation de la mission est également conditionnée par les horaires de marée, la basse mer permettant d'acquérir des données sur l'estran.

Des cibles circulaires rouges de $25 \mathrm{~cm}$ de diamètre sont disposées sur le sol. La densité de ce semis de cibles est ajustée en fonction de la couverture au sol d'une image afin qu'une dizaine de cibles soit visible sur un cliché. La position exacte du centre de chaque cible est mesurée au GPS différentiel en mode RTK avec une précision centimétrique.

Le plan de vol de DRELIO est alors programmé dans le logiciel de pilotage automatique, puis transféré à l'auto-pilote de l'hélicoptère (voir figure 2). Ce plan de vol inclut le point de décollage, les points de passage définissant la trajectoire, le point d'atterrissage, la vitesse de l'hélicoptère (généralement de l'ordre de $5 \mathrm{~km} \mathrm{~h}^{-1}$ ) et le lieu d'atterrissage d'urgence en cas de perte du lien radio. L'altitude de vol de DRELIO est généralement comprise entre 50 et $200 \mathrm{~m}$. Avec un objectif de focale de 35 ou $50 \mathrm{~mm}$, la taille du pixel est alors de l'ordre de $2 \mathrm{~cm}$ pour une couverture de $34 \times 22 \mathrm{~m}$ à $135 \times 90 \mathrm{~m}$. Après l'atterrissage, les clichés sont transférés de l'appareil photographique à un ordinateur. 


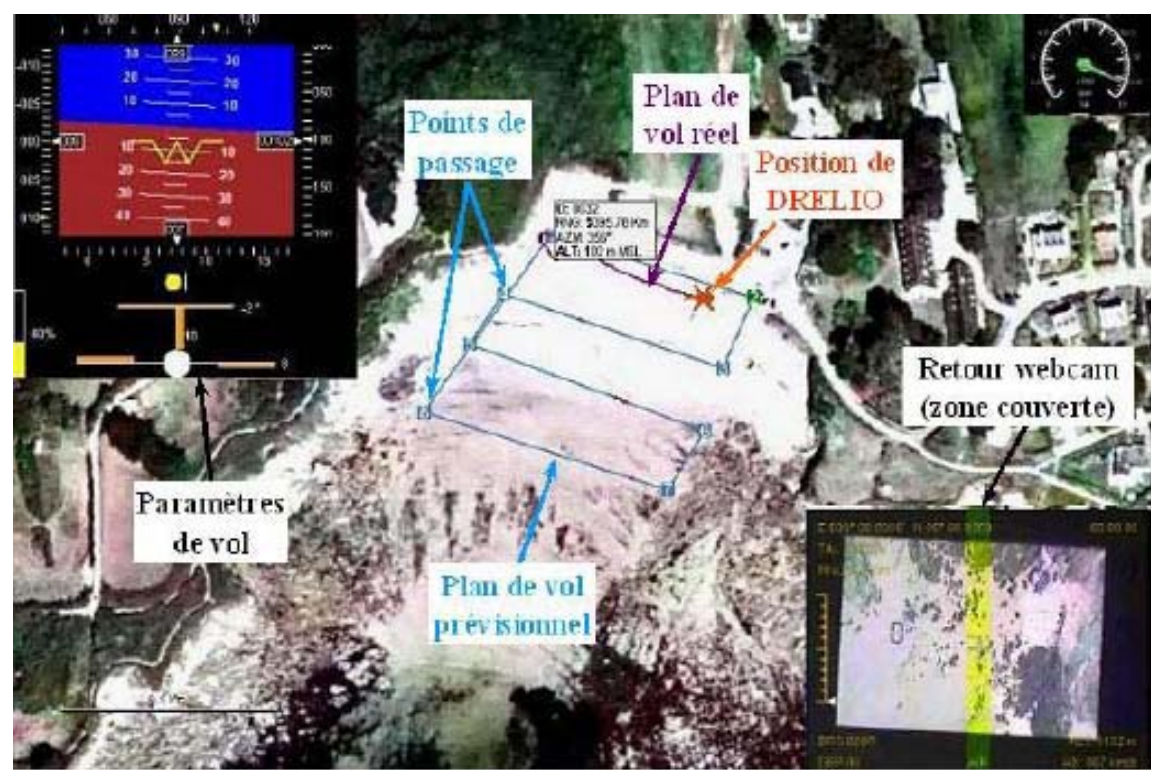

Figure 2. Ecran de contrôle de l'auto-pilote.

\section{Génération de MNT}

Deux images d'une même zone acquises successivement sous des angles légèrement différents dans des conditions reproduisant des conditions stéréoscopiques permettent de générer des Modèles Numériques de Terrain (MNT) par stéréo-photogrammétrie. Le taux de recouvrement de ces images doit être de l'ordre de $60 \%$.

Pour mettre en œuvre cette méthode, deux types de paramètres doivent être déterminés : - les caractéristiques optiques de l'appareil photographique (paramètres internes).

- la position et l'orientation de l'appareil photographique au moment de la prise de vue (paramètres externes).

Les paramètres internes sont en partie fournis par le fabricant de l'appareil photographique (la distance focale de la lentille $(c)$, les coordonnées $\left(\eta_{0}, \xi_{0}\right)$ du point principal dans le repère image qui correspond à la projection de l'axe optique sur l'image) ou doivent être calculés avant la mission via une calibration car variables dans le temps (paramètres de distorsion de la lentille - KASSER \& EGELS, 2002).

Les paramètres externes de l'appareil photographique sont recalculés pour chaque image. Ce sont les coordonnées $\left(X_{0}, Y_{0}, Z_{0}\right)$ du point principal de l'appareil et les angles $(\omega, \phi, \kappa)$ de l'orientation de l'axe optique (voir figure 3 ).

Bien que théoriquement similaires aux techniques de stéréophotogrammétrie conventionnelles appliquées sur les images acquises lors de campagnes aériennes (BOUILLON et al., 2006), la technique de restitution de la topographie à partir d'images acquises par drone nécessite des développements complémentaires notamment pour la restitution précise des paramètres internes et externes..

En effet, pour des raisons de coût et de masse embarquée, Drelio est équipé d'un appareil réflex numérique "grand public" et non d'une chambre photogrammétrique. De 
plus, afin d'optimiser la durée d'une mission et de réduire le nombre d'images nécessaires pour couvrir une zone donnée, des lentilles à petite distance focale $(35 \mathrm{~mm}$ contre $135 \mathrm{~mm}$ ) sont utilisées (KASSER \& EGELS, 2002). La maximisation de la surface couverte au sol s'accompagne de distorsions optiques plus importantes. Ces distorsions correspondent aux écarts géométriques entre une lentille "parfaite" et une lentille réelle, écarts qui dépassent parfois les 5 pixels sur le bord des clichés pour l'appareil photo embarqué sur DRELIO.

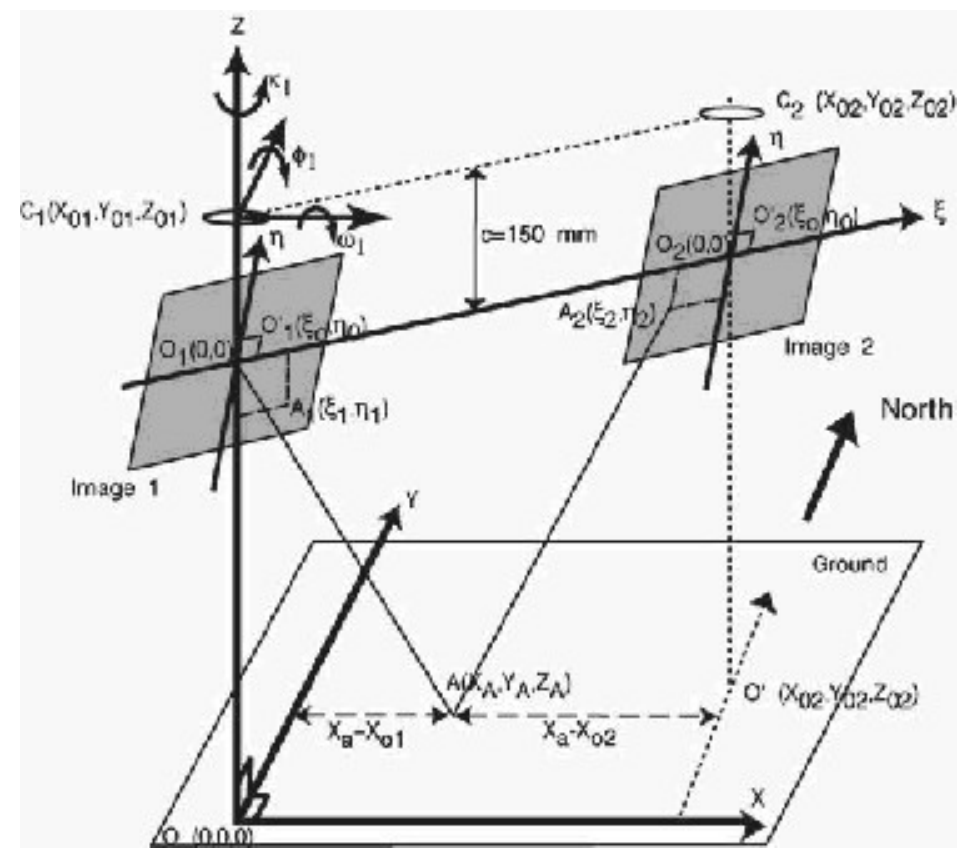

Figure 3. Systèmes de référence et caractéristiques géométriques d'un couple stéréoscopique (d'après KRAUS et al., 1979; JENSEN, 2000 ; CASSON et al., 2003)

$(X, Y, Z)$ : système de référence absolu ; $C_{1}\left(X_{01}, Y_{01}, Z_{01}\right)$ et $C_{2}\left(X_{02}, Y_{02}, Z_{02}\right)$ : positions 1 et 2 de la caméra dans le repère absolu de référence; $O^{\prime}$ : projection verticale de $C_{2}$ sur le sol; $(\eta, \xi)$ : repère "image" de référence; $O_{1}(0,0)$ et $O_{2}(0,0)$ : centres respectifs des clichés; $O_{1}{ }^{\prime}$ et $O_{2}{ }^{\prime}$ : intersections réelles axes optiques-capteur (points principaux); $A_{1}$ et $A_{2}$ : points "images" correspondant au point au sol $A ;\left(X_{a}\right.$

$\left.-X_{01}\right)$ : distance $A-C_{1}$ selon l'axe des $X ;\left(X_{a}-X_{02}\right): A$ - $C_{2}$ selon l'axe des $X ; c$ : distance focale de l'objectif; $(\omega, \phi, \kappa)$ : angles de rotation de la camera.

L'utilisation d'une petite plate-forme telle que DRELIO complexifie la restitution des paramètres externes. En effet, en raison de sa faible vitesse et de sa faible masse, la trajectoire de DRELIO n'est pas parfaitement stable. De plus, les contraintes techniques ne permettant pas d'équiper le drone d'instruments de positionnement suffisamment précis, les paramètres externes sont donc mal connus a priori. La restitution de ces paramètres est réalisée via un jeu de points remarquables repérés sur les 2 images 
(coordonnées images) et dont les coordonnées terrains sont calculées dans un repère lié à la caméra 1 : les GCP (Ground Control Points) "relatifs".

Connaissant la position au sol des GCP relatifs ainsi que leurs coordonnés images, une première DLT (Direct Linear Transformation), (ABDEL-AZIZ \& KARARA, 1971 ; MIKHAIL et al., 2001) permet d'estimer approximativement la position et l'orientation $(\mathrm{X}, \mathrm{Y}, \mathrm{Z}, \omega, \phi, \kappa)$ de la caméra au moment de l'acquisition de chacun des deux clichés. Cette solution approximée est ensuite utilisée comme valeur de départ dans un processus de minimisation au sens des moindres carrés qui permet récursivement de préciser la position au sol des GCP relatifs et de restituer rigoureusement les positions et orientations des caméras. La précision du positionnement final des caméras est inférieure à $2 \mathrm{~cm}$ en planimétrie et inférieure à $10 \mathrm{~cm}$ en vertical.

Les deux images du couple stéréoscopique sont ensuite projetées dans une géométrie proche "pseudo-orthorectifiée", se prêtant à une comparaison par fenêtre glissante.

L'étape suivante consiste, par corrélation d'images, à déterminer la position de chaque point de la première image pseudo-orthorectifiée et la position du point correspondant sur la seconde image pseudo-orthorectifiée (BARATOUX et al., 2001 ; DELACOURT et al., 2004). On obtient finalement trois matrices contenant pour chaque point le coefficient de corrélation et les décalages respectifs selon $\mathrm{x}$, et y entre les deux images pseudo-orthorectifiées.

Le résultat de la corrélation est validé si le coefficient de corrélation dépasse le seuil fixé. Si ce seuil est élevé, le nombre de points corrélés est faible mais la qualité de la corrélation est très bonne. Inversement, si ce seuil est faible, il y aura beaucoup de points corrélés mais certains points pourront être mal appariés.

Enfin, les équations de la photogrammétrie sont appliquées à chaque paire de points homologues en coordonnées image afin de retrouver leurs coordonnées 3D (KRAUS \& WALDHÄUSL, 1994) en minimisant la distance entre les lignes de visée de chaque pixel. La distance minimale entre les lignes de visée (égale à zéro dans un cas idéal) est une mesure de la précision du MNT généré. Dans un premier temps, ce MNT est construit dans le repère relatif lié à la caméra 1 . Il est ensuite projeté dans un repère géographique absolu grâce aux cibles géoréférencées. A partir du MNT, il est enfin possible de construire les images corrigées de la distorsion due au relief, qualifiées d'"images orthorectifiées".

\section{Résultats}

Une acquisition a été réalisée sur la Plage de Porsmilin dans l'anse de Bertheaume, située à l'entrée de la Rade de Brest. La mission a été effectuée au mois de juin 2009. L'altitude de vol était proche de $100 \mathrm{~m}$ et la focale de l'objectif était de $35 \mathrm{~mm}$, ce qui implique une fauchée au sol de $67 \times 45 \mathrm{~m}$ pour une résolution inférieure à $2 \mathrm{~cm}$. 85 cibles ont été réparties sur la plage et positionnées au DGPS (Differential Global Positioning System) avec une précision centimétrique et 266 images ont été acquises. 
En parallèle, le laboratoire Géomer a également acquis des mesures DGPS sur l'ensemble de la plage, à raison d'un point tous les $2 \mathrm{~m}$ en moyenne. Un MNT a été généré en interpolant directement ces points DGPS.

Parmi les clichés acquis, une paire d'images stéréoscopiques a été sélectionnée. Grâce à la chaîne de calcul décrite précédemment, une ortho-image et un MNT avec une résolution spatiale de $4 \mathrm{~cm}$ ont été générés (voir figure 4) et ont pu être insérés dans un SIG (Système d'Information Géographique) (voir figure 5). Un profil transversal en a été extrait (voir figure 6). Il montre une morphologie caractéristique de houle de beau temps marquée par la présence d'une berme en haut de plage et d'une absence de formes intertidales en bas de plage.

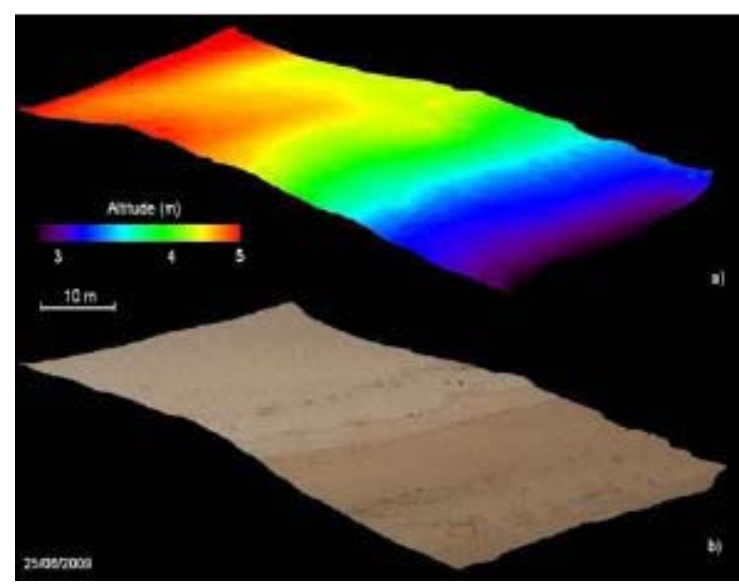

Figure 4. représentation $3 D$ (exagération 4) du MNT (a) et ortho-image (b) générés à partir d'un couple d'image acquises lors de la mission Porsmilin du 25/06/2009

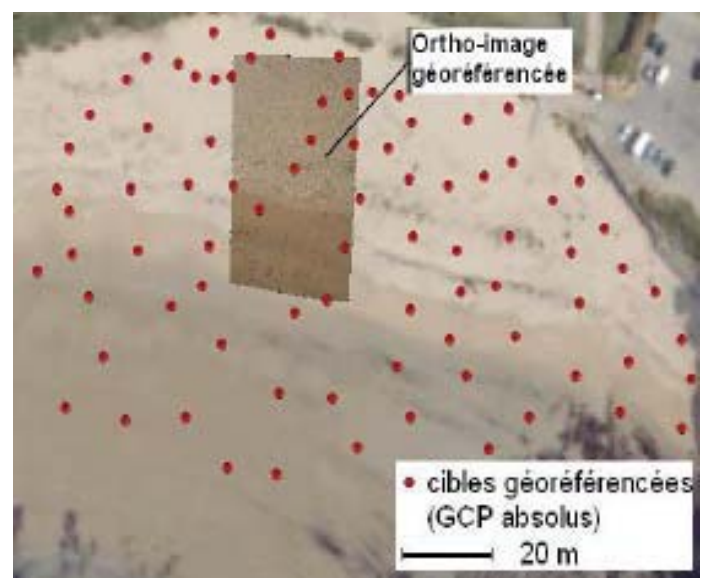

Figure 5. Ortho-image géoréférencé insérée dans un SIG.

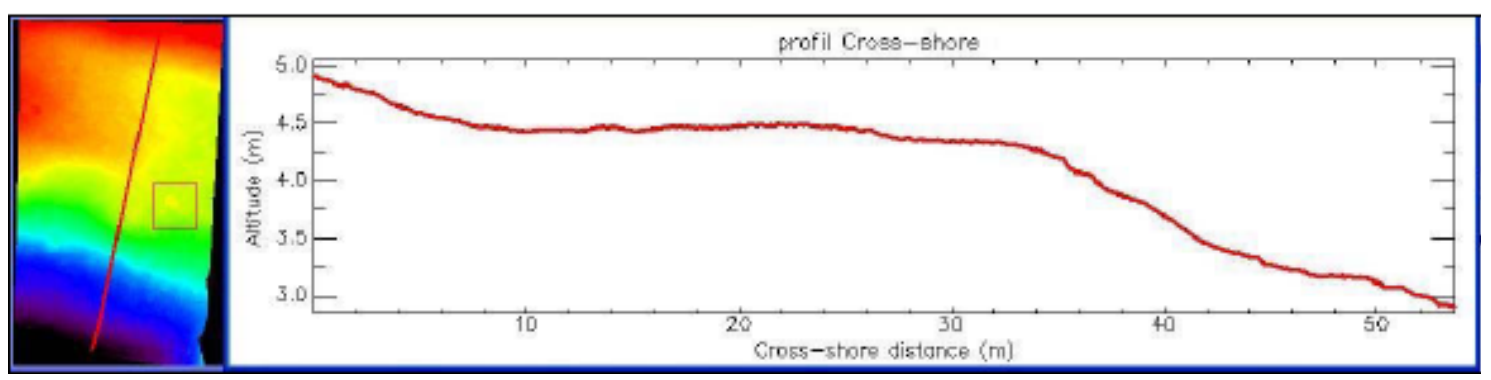

Figure 6. Profil transversal selon Z sur le MNT généré précédemment.

Le MNT contient des petits détails de la topographie. Par exemple, une "plate-forme" de sable sans doute aménagée par des enfants sur environ $1,5 \mathrm{~m}$ de diamètre et $10 \mathrm{~cm}$ de hauteur (correspondant vraisemblablement à un "château de sable") est visible sur les profils topographiques extraits du MNT (voir figure 7). 
La qualité des MNT drone a été estimée en comparant la position des cibles mesurée au DGPS et leur position sur le MNT géoréférencé. En $(x, y)$, la précision est de moins de $10 \mathrm{~cm}$ et la précision selon $\mathrm{z}$ est de $+/-8 \mathrm{~cm}$. Il s'agit là d'une erreur moyenne, prenant en compte les mesures d'erreur y compris sur les cibles situées dans des zones mal corrélées où le calcul du MNT résulte d'une interpolation et est donc moins précis.

Ces données ont également été comparées au MNT global généré à partir du levé au DGPS. L'écart vertical moyen entre les données du MNT au DGPS et les données du MNT calculé par photogrammétrie est en moyenne de $+/-15 \mathrm{~cm}$ (voir figure 8 ).

\section{Discussions et conclusions}

La principale difficulté à l'utilisation de DRELIO pour la génération de MNT par stéréophotogrammétrie réside dans la précision de son positionnement. Les cibles au sol géoréférencées sont donc nécessaires, alors que leur installation et la mesure de leur position est l'étape la plus coûteuse en terme de temps sur le terrain. Une perspective d'amélioration consiste donc à équiper DRELIO de nouveaux capteurs permettant une mesure suffisamment précise des paramètres externes ce qui permettrait de s'affranchir de la présence de cibles.

Le tableau 1 récapitule les principaux avantages de DRELIO sur les autres platesformes télécommandées.

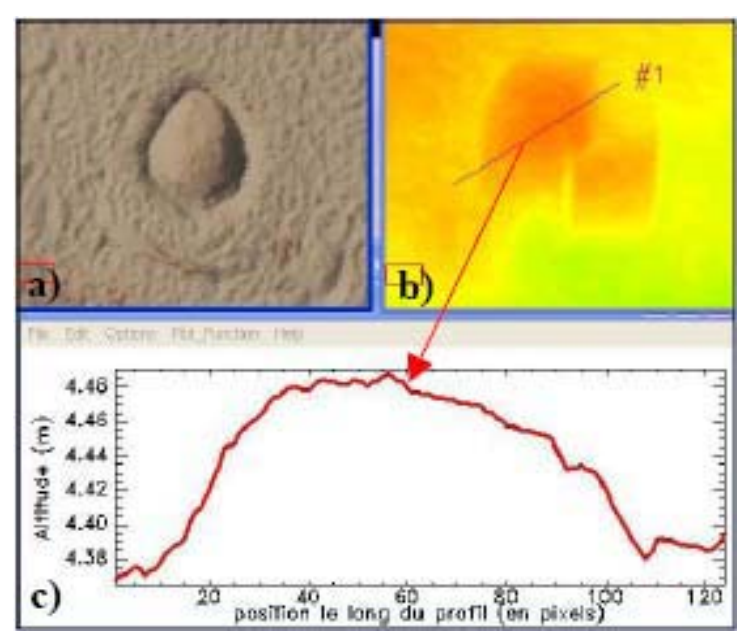

Figure 7. Zoom sur un détail: une plateforme de sable d'environ 1,5 $\mathrm{m}$ de diamètre visualisée sur l'ortho-image (a), le MNT

(b) et le profil en coupe verticale (c).

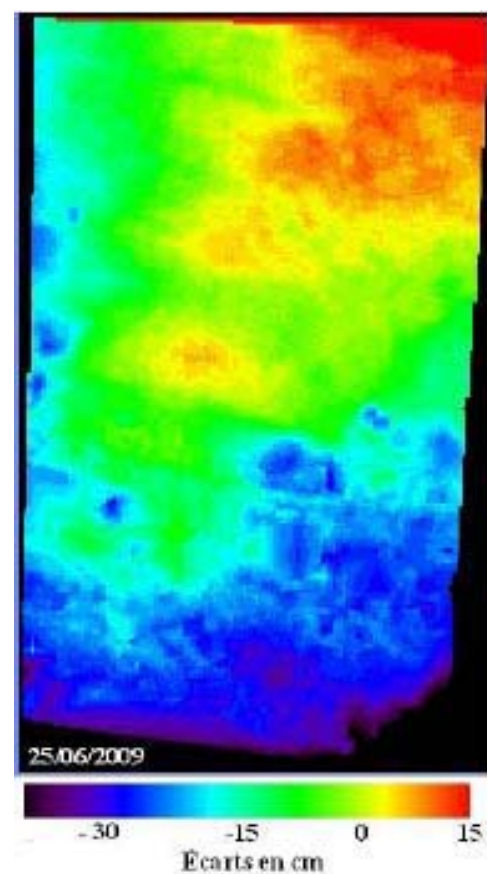

Figure 8. MNT différentiel entre le MNT réalisé à partir du levé DGPS et le MNT généré par stéréorestitution. 
Le LIDAR aéroporté ou le TLS (Terrestrial Laser Scanner) sont deux systèmes d'acquisition dont les résultats sont comparables en terme de précision aux MNT issus d'images DRELIO. Toutefois, l'utilisation d'un LIDAR aéroporté est très coûteuse et, étant données les contraintes des plates-formes sur lesquelles il peut être embarqué, la résolution n'est que métrique. Le TLS atteint quant à lui une résolution centimétrique, mais étant donnée la faible topographie de la plage, plusieurs mises en station seront nécessaires pour couvrir complètement la zone, ce qui peut s'avérer assez long. De plus, le LIDAR et le TLS sont des systèmes très onéreux.

Tableau 1. Inconvénients majeurs des autres plates-formes télécommandées par rapport à DRELIO.

\begin{tabular}{ll}
\hline Type de plateforme & Inconvénients par rapport à DRELIO \\
\hline hélicoptères à moteur électrique & charge utile trop faible - autonomie $<15$ min \\
avion à moteur thermique & évolution difficile sur de petites surfaces (décollage, \\
& virages, atterrissage) \\
drone paramoteur & trop sensible aux conditions aérologiques \\
& vitesse et altitude de survol trop élevées (réglementation) \\
avion / hélicoptère * & pour de la haute résolution - coût de l'heure de vol \\
\hline
\end{tabular}

Dans ce contexte, le système DRELIO présente pour sa part une grande flexibilité d'utilisation. En effet, il n'a aucune contrainte de vitesse pour les acquisitions (aptitude au vol stationnaire), ce qui offre une grande netteté d'image. Étant envisageables tout au long de l'année, les missions peuvent être mises en œuvre par deux ingénieurs, avec une logistique très simple en moins d'un jour, et les résultats sont disponibles en quelques heures.

Les images obtenues permettent de générer des MNT de $4 \mathrm{~cm}$ de résolution spatiale avec une précision égale à deux fois la résolution sur une zone couverte de $67 \mathrm{~m} \times 45 \mathrm{~m}$. En outre, le large potentiel de ce système n'a pas encore été complètement exploité. Les images acquises par DRELIO peuvent également être utilisées pour générer des MNT des zones faiblement immergées (fond visible sous la tranche d'eau) soit par des méthodes de photogrammétrie classique, soit à partir de la couleur de l'eau (LEJOT et al., 2007). Le suivi multi-temporel de zones affectées par des processus modifiant la topographie (tempêtes, crues, ..) permet de construire les MNT successifs, de les comparer les uns aux autres et donc de quantifier les transferts de sédiments sur le cordon littoral. 


\section{Remerciements :}

Ces travaux sont financés par le CNRS / INSU Programme Relief, le CNES et la région Bretagne.

\section{Références bibliographiques}

ABDEL-AZIZ Y.I., KARARA H.M. (1971). Direct linear transformation into object space coordinates in closerange photogrametry. Proc. Symp. Close-Range Photogrametry, pp 1-18.

BARATOUX D., DELACOURT C., ALLEMAND P. (2001). High Resolution Digital Elevation Model derived from Viking images : New method and comparison with MOLA data. Journal of Geophysical Research, 106, pp 32927.

BOUILLON A., BERNARD M., GIGORD P., ORSONI A., RUDOWSKI V., BAUDOIN A. (2006). SPOT 5 HRS geometric performances: Using block adjustment as a key issue to improve quality of DEM generation. Isprs Journal of Photogrammetry and Remote Sensing, v. 60, pp 134-146. doi:10.1016/j.sprspprs.2006.03.002

CASSON B., DELACOURT C., ALLEMAND P. (2005). Contribution of multitemporal remote sensing images to characterize landslide slip surface - Application to the La Clapière landslide (France). Natural Hazards and Earth System Science, 5, pp 425-437.

DELACOURT C., ALLEMAND P., CASSON B., VADON H. (2004). Velocity field of the "La Clapière" landslide measured by the correlation of aerial and QuickBird images. Geophysical Research Letter, L15619, 31 p.

DELACOURT C., ALLEMAND P., JAUD M., GRANDJEAN P., DESCHAMPS A., AMMANN J., CUQ V., SUANEZ S. (2009). DRELIO: An Unmanned Helicopter for Imaging Coastal Areas. Journal of Coastal Research 2, pp 1489-1493.

DEKKER A.G., BRANDO V.E., ANSTEE J.M., PINNEL N., KUTSER T., HOOGENBOOM H.J., PASTERKAMP R., PETERS S.W.M., VOS R.J., OLBERT C., MALTHUS T. J. (2001). Imaging spectrometry of water, Ch. 11 in: Imaging Spectrometry: Basic principles and prospective applications: Remote Sensing and Digital Image Processing, Dordrecht, Kluwer Academic Publishers v.IV, pp 307-359.

HAMM L., CAPOBIANCO M., DETTE H.H., LECHUGA A., SPANHOFF R., STIVE M.J.F. (2002). A summary of European experience with shore nourishment. Coastal Engineering, 47(2), pp 237-264. doi:10.1016/80378-3839(02)00127-8

JACOME A. (2009). MNT à très haute résolution spatiale pour la représentation $3 D$ de ravines d'érosion en montagne. Thèse, Université de Montpellier II, 216 p.

JENSEN J.R. (2000). Processing Remotely Sensed Data: Hardware and Software Considerations. Remote Sensing in Hydrology and Water Management, SCHULTZ G. A. and ENGMAN E.T. (Eds.), Berlin: Springer-Verlag, pp 49-59

KASSER M, EGELS Y. (2002). Digital Photogrammetry. Taylor and Francis: London. $351 \mathrm{p}$. 
Thème 3 - Instrumentation, mesures, imagerie et télédétection

KRAUS K, WALDHAÜSL P. (1994). Photogrammetry, Fundamentals and Standard Processes. 4th ed.- vol. 1 Dummler ISBN 3-427-78684-6, 397 p.

LEJOT J., DELACOURT C., PIEGAY H, FOURNIER T, TREMELO M.L., ALLEMAND P. (2007). Very high spatial resolution imagery for channel bathymetry and topography from an unmanned mapping controlled plateform. Earth Surface Processes and Landforms, 32 (11), pp 1705-1725. doi:10.1002/esp.1595

MEUR-FÉREC C., DEBOUDT P., MOREL V. (2008). Coastal risks in France: an integrated method for evaluating vulnerability. Journal of Coastal Research, 24(2B), pp 178-189. doi:10.2112/05-0609.1

MIKHAIL E., BETHEL J., MCGLONE J. (2001). Introduction to Modern Photogrammetry. John Wiley and Sons (WIE), 479 p.

RIEB G., WLAKER P. (2001). Suivi morphologique du littoral. Géologues, 129, pp 75-79.

RUGGIERO P., VOIGT B., KAMINSKY G. (2000). Beach monitoring for enhanced decision-making, Coastal Society 17th Conference Coasts at the Millennium - Portland, Oregon, pp 516-524.

SOLOMON S.M., FORBES D.L. (1999). Coastal hazards and associated management issues on South Pacific Islands. Ocean \& Coastal Management, 42, pp 523-554. doi:10.1016/S0964-5691(99)00029-0

SUGIURA R., NOGUCHI N., ISHII K. (2005). Remote-sensing technology for vegetation monitoring using an unmanned helicopter. Biosystems engineering, 90-4, pp 369-379. doi:10.1016/j.biosystemseng.2004.12.011 